\title{
Bacterial cyanogenesis occurs in the cystic
} fibrosis lung

\author{
K. Sanderson, L. Wescombe, S.M. Kirov, A. Champion and D.W. Reid
}

ABSTRACT: The cystic fibrosis (CF) lung environment is poorly defined, but data suggest that bacteria may encounter reduced oxygen tensions and possibly an anaerobic environment. Pseudomonas aeruginosa produces the potent toxin cyanide under strictly microaerobic conditions. Evidence of bacterial cyanogenesis in the CF lung was investigated in the present study by measuring sputum cyanide concentrations.

Sputum cyanide was measured in seven stable CF patients, as well as before and after intravenous antibiotic therapy during a hospital admission in a further eight patients experiencing acute exacerbations. All patients were chronically infected with $\boldsymbol{P}$. aeruginosa. Comparative sputum data were obtained from nine CF patients with no documented $P$. aeruginosa infection and 10 healthy, nonsmoking normal controls.

High levels of cyanide were detected in all the $P$. aeruginosa-infected stable CF patients (median (range) $\left.0.56(0.37-2.81) \mu \mathrm{g} \cdot \mathrm{mL}^{-1}\right)$, and in seven out of eight acute sputum samples $(0.73$ $\left.(0-1.43) \mu \mathrm{g} \cdot \mathrm{mL}^{-1}\right)$. In contrast, cyanide was not detectable in sputum from eight out of nine CF patients without $\boldsymbol{P}$. aeruginosa infection or in any of the normal controls. Intravenous antibiotic treatment significantly reduced sputum cyanide levels (median 0.73 to median $0.0 \mu \mathrm{g} \cdot \mathrm{mL}^{-1}$ ).

The cyanide detected indicates that the cystic fibrosis lung provides a predominantly microaerobic environment for Pseudomonas aeruginosa. Cyanide is likely to be a potentially important virulence factor in Pseudomonas aeruginosa-infected cystic fibrosis patients.

KEYWORDS: Cyanide, microaerobic, Pseudomonas aeruginosa, pulmonary disease

I $\mathrm{n}$ individuals with cystic fibrosis (CF) and other destructive lung diseases, Pseudomonas aeruginosa is an opportunistic bacterium that causes chronic airway sepsis. In CF, it becomes the predominant pathogen by late childhood [1]. This early acquisition of $P$. aeruginosa in relatively normal lungs is unique to CF. Much of the lung damage that occurs in $\mathrm{CF}$ is thought to be due to ineffective host factors [2]. Bacterial virulence factors undoubtedly also contribute to lung damage, but they have been poorly characterised in vivo. In CF airways, $P$. aeruginosa is thought to grow in anaerobic/reduced oxygen pockets situated within thickened and tenacious mucus plugs that occlude small airways [3,4].

This environment may be suitable for cyanide production by $P$. aeruginosa [5-7]. However, to date, it is not known whether bacteria are residing in strictly anaerobic or microaerobic pockets within the CF lung. $P$. aeruginosa is one of only a few bacterial species ( $P$. fluorescens, Chromobacterium violaceum and Rhizobium leguminosarum) known to produce cyanide [8]. The $P$. aeruginosa cyanide synthase enzyme is cell associated and requires molecular oxygen as an electron acceptor. Cyanide is only produced over a very narrow spectrum of environmental oxygen tensions (microaerobic) and synthesis is rapidly inactivated under both atmospheric oxygen and strictly anaerobic conditions [8]. Therefore, cyanide levels were measured in sputum samples from CF patients as both an in vivo marker of $P$. aeruginosa microaerobic growth and to determine if cyanide production could be a potential virulence mechanism in CF lung disease.

\section{METHODS}

The Southern Tasmania Health and Medical Human Research Ethics Committee approved the study and all subjects gave written informed consent before participating. In total, 15 adult CF patients with chronic $P$. aeruginosa lung infection (confirmed by repeated routine microbiological testing) were recruited at the time of a routine clinic appointment for stable patients $(\geqslant 1$ month from most recent exacerbation), or when unwell with worsening cough, breathlessness and sputum purulence and admitted to hospital (acute patients). In the acute patients, a repeat sputum sample was obtained $\geqslant 1$ week into the intravenous antibiotic course (median (range) 8.5
AFFILIATIONS

Respiratory Research Group, Menzies Research Institute, University of Tasmania, Hobart, Australia.

CORRESPONDENCE

D.W. Reid

School of Medicine

University of Tasmania

43 Collins Street

Hobart

TAS

Australia 7001

Fax: 61362264894

E-mail: d.e.c.reid@utas.edu.au

Received:

November 132007

Accepted after revision:

April 162008

\section{SUPPORT STATEMENT}

The present study received support from the Institutional Research Grant Scheme, University of Tasmania

(Hobart, Australia).

STATEMENT OF INTEREST

None declared. 
TABLE 1 Subject demographics

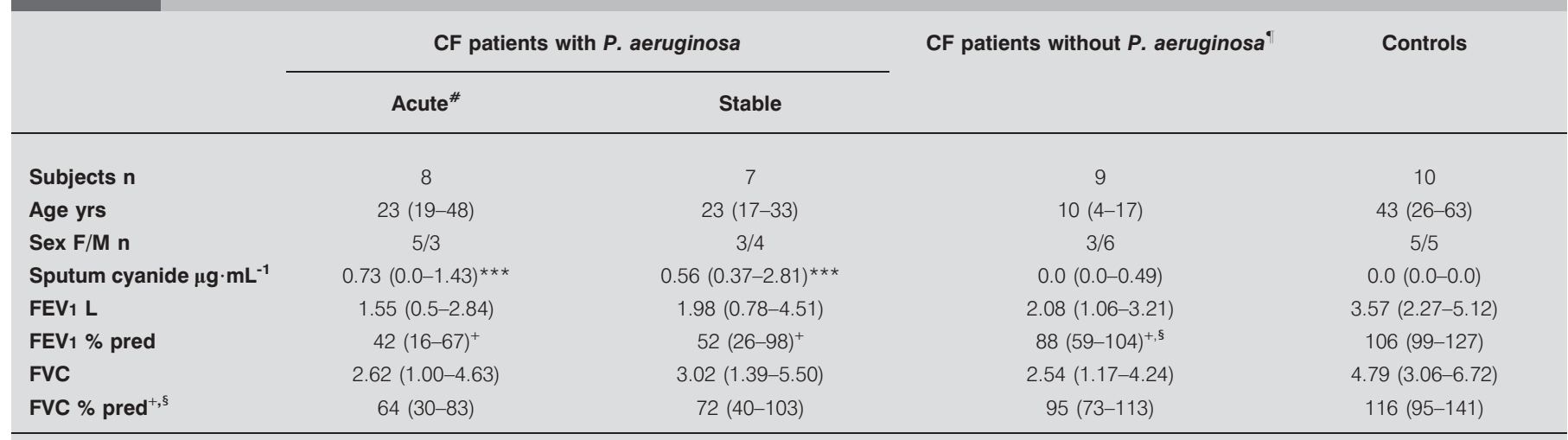

Data are presented as median (range), unless otherwise stated. CF: cystic fibrosis; $P$. aeruginosa: Pseudomonas aeruginosa; F: female; M: male; FEV1: forced expiratory volume in one second; \% pred: \% predicted; FVC: forced vital capacity. " : lung function values achieved within $48 \mathrm{~h}$ of admission; ": Iung function results are based on seven patients, as two patients aged 4 and 5 yrs could not manage reproducible spirometry; ${ }^{+}$: CF patients versus healthy controls, $p<0.01 ;{ }^{s}$ : $P$. aeruginosa-infected (stable and acute patients) versus $C F$ patients without $P$. aeruginosa infection, $p<0.01$. ${ }^{* *}$ : $P$. aeruginosa-infected (stable and acute patients) versus cystic fibrosis (CF) patients without $P$. aeruginosa infection and healthy controls, $p<0.001$

(7-14) days) at a time-point when symptoms were subjectively improving. In total, 14 patients were receiving long-term anti- $P$. aeruginosa antibiotic treatment regimes by inhalation (data not shown). One patient was not receiving any form of antibiotic therapy directed against $P$. aeruginosa at the time of sampling. Nine patients who had not grown $P$. aeruginosa previously also provided sputum samples. Spontaneously expectorated sputum was collected and processed as described previously [9]. Induced sputum samples were obtained from 10 healthy volunteers using the method of PIN et al. [10] and processed in the same way. All CF patients and normal controls were current nonsmokers.

\section{Sputum processing}

An aliquot of raw sputum was weighed and an equivalent volume to weight of dithiothreitol $(10 \%)$ was added. The sample was gently vortexed and placed in a water bath $\left(38^{\circ} \mathrm{C}\right)$ for $30 \mathrm{~min}$. At 10-min intervals the sample was removed and gently vortexed again. If the sample appeared particularly tenacious, further mixing was undertaken with gentle pipetting to ensure homogenisation. The sample was diluted a further five times with PBS, ensuring a constant final dilution effect of 10 times, and then centrifuged at $350 \times g$ for $15 \mathrm{~min}$. Following centrifugation, the cell-free supernatant was decanted and stored at $-80^{\circ} \mathrm{C}$ in $1-\mathrm{mL}$ aliquots for further analysis.

\section{Cyanide detection}

Cyanide was liberated from the processed sputum supernatants by acid treatment and assayed using a standard method (4500-CN; American Public Health Association, Washington, DC, USA). The lower limit of detection of the method was $0.05 \mu \mathrm{g} \cdot \mathrm{L}^{-1}$.

\section{Statistical analysis}

Differences between groups were assessed using the KruskalWallis test for nonparametric data. The Wilcoxon's rank test was used to compare sputum cyanide levels in samples obtained at the start of an exacerbation and at the end of an intravenous antibiotic treatment course. A two-tailed p-value of $\leqslant 0.05$ was considered to be statistically significant.

\section{RESULTS}

Healthy controls were significantly older and had better lung function than the CF patients (table 1). Not surprisingly, CF patients without $P$. aeruginosa infection were younger and had better lung function than those with chronic $P$. aeruginosa infection. Cyanide was present in all of the sputum samples from stable CF patients with $P$. aeruginosa (median (range) 0.56 $\left.(0.37-2.81) \mu \mathrm{g} \cdot \mathrm{mL}^{-1}\right)$, and in seven out of eight samples from acute patients $\left.0.73(0.0-1.43) \mu \mathrm{g} \cdot \mathrm{mL}^{-1}\right)$. There was no statistical difference between sputum cyanide levels in acute and stable patients $(p=0.9)$. Cyanide was not detected in eight out of nine sputum samples from CF patients without $P$. aeruginosa or in

\begin{tabular}{cccc} 
TABLE 2 & $\begin{array}{l}\text { Demographic details and sputum culture results } \\
\text { in stable cystic fibrosis patients without } \\
\text { Pseudomonas aeruginosa infection }\end{array}$ \\
Subject & Age yrs Sex & Sputum microbiology \\
\hline $\mathbf{1}^{\#}$ & 3 & M & S. aureus \\
$\mathbf{2}$ & 16 & M & MRSA, S. prolificans \\
$\mathbf{3}$ & 15 & M & S. aureus, A. fumigatus \\
$\mathbf{4}$ & 10 & F & S. aureus, H. influenzae \\
$\mathbf{5}$ & 16 & F & S. aureus \\
$\mathbf{6}$ & 12 & M & S. aureus, A. xylosoxidans, S. prolificans \\
$\mathbf{7}$ & 8 & M & A. fumigatus \\
$\mathbf{8}$ & 4 & M & S. aureus, H. influenzae, A. fumigatus, S. maltophilia \\
$\mathbf{9}$ & 6 & F & S. aureus \\
\hline
\end{tabular}

M: male; F: female; S. aureus: Staphylococcus aureus; MRSA: methicillinresistant Stapylococcus aureus: S. prolificans: Scedosporium prolificans; A. fumigatus: Aspergillus fumigatus; $H$. influenzae: Haemophilus influenzae; A. xylosoxidans: Alcaligenes xylosoxidans; S. maltophilia: Stenotrophomonas maltophilia. ${ }^{*}$ : the only patient with detectable sputum cyanide levels. 
any of the sputum samples from normal controls (table 1 and fig. 1). The only CF patient without $P$. aeruginosa who had detectable sputum cyanide was $<5$ yrs of age. This patient had isolated Staphylococcus aureus in the only two sputum samples obtained to date (table 2).

Intravenous antibiotic treatment for an acute exacerbation reduced sputum cyanide levels (median (range) 0.73 (0.01.43) $\mu \mathrm{g} \cdot \mathrm{mL}^{-1}$ to $\left.0.0(0.0-1.00) \mu \mathrm{g} \cdot \mathrm{mL}^{-1}, \mathrm{p}=0.05\right)$. In four of the seven acute patients with detectable cyanide levels on admission, the levels were reduced to below the detection limit of the assay following intravenous antibiotic treatment.

Interestingly, the highest sputum cyanide concentration was found in a stable patient who was the only individual chronically infected with $P$. aeruginosa and not receiving any form of routine antibiotic therapy directed against the bacterium. There was no relationship between sputum cyanide levels and lung function.

\section{DISCUSSION}

This is the first report of cyanide in CF sputum and the present results support previous speculation that the $C F$ lung environment may be suitable for $P$. aeruginosa cyanogenesis. Both clinically stable CF patients and those presenting with acute exacerbations of $\mathrm{CF}$ had high levels of cyanide, whereas cyanide was undetectable in sputum samples from normal healthy controls and nearly all sputum samples obtained from CF patients from whom $P$. aeruginosa had not previously been isolated. Following intravenous antibiotic treatment for an acute exacerbation, sputum cyanide levels were significantly reduced in patients infected with $P$. aeruginosa.

Cyanide is a very potent poison that causes cell death through irreversible inhibition of mitochondrial oxidative phosphorylation. Cyanide toxicity is well described following smoke inhalational injuries in humans, but the role of cyanide in human disease in the setting of $P$. aeruginosa infection is poorly characterised. However, cyanide production has been noted in $P$. aeruginosa-infected burn eschars [11-13]. The cellular toxicity of cyanide is greatly influenced by the chemical milieu but, in humans, blood cyanide levels of $0.5-1.0 \mu \mathrm{g} \cdot \mathrm{mL}^{-1}$ are associated with cardiac effects, levels of $2.5-3.0 \mu \mathrm{g} \cdot \mathrm{mL}^{-1}$ result in reduced consciousness and levels $>3.0 \mu \mathrm{g} \cdot \mathrm{mL}^{-1}$ cause death [14]. Therefore, the sputum levels detected in the present study are well within the toxic range for cell metabolism and will probably contribute to long-term lung damage in $\mathrm{CF}$.

The capacity of $P$. aeruginosa to produce cyanide has been recognised for some time [12]. Most of the current understanding of the genetics, biochemistry and regulation of cyanide production by $P$. aeruginosa comes from studying its interactions in soil communities, where $P$. aeruginosa uses cyanide to protect its niche and eradicate competing organisms [8]. The current finding of high cyanide levels in CF sputum has several disease implications. First, cyanide may be used by $P$. aeruginosa in the lung in the same way that it is utilised in soil, that is, to protect its niche. This may partly explain why $P$. aeruginosa rapidly becomes the predominant pathogen in $\mathrm{CF}$, apparently displacing other bacterial species. Secondly, cyanide will be directly toxic to airway cells and, finally, cyanide may also contribute to the relative inability of neutrophils to clear $P$. aeruginosa infection in the CF lung $[15,16]$. In the

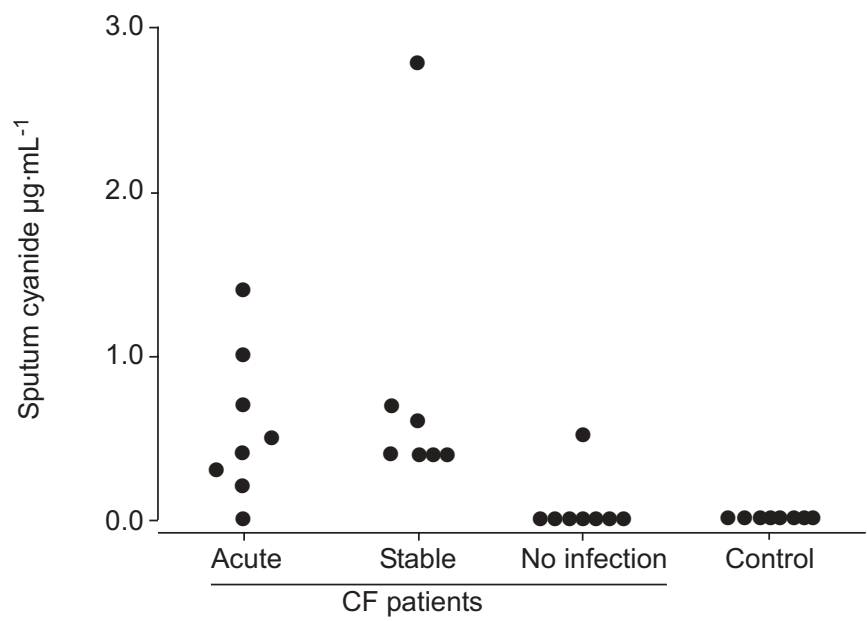

FIGURE 1. Cyanide concentrations in sputum samples from cystic fibrosis (CF) patients with chronic Pseudomonas aeruginosa infection experiencing an acute exacerbation $(n=8)$, stable CF patients with chronic $P$. aeruginosa infection $(n=7)$, stable CF patients with no documented $P$. aeruginosa infection $(n=9)$ and healthy controls $(n=10)$. Each point represents one sputum sample.

concentrations reported, cyanide has been shown to inhibit the function of the enzyme myeloperoxidase and prevent production of hypochlorous acid during the oxidative burst [17]. Cyanide may also impair neutrophil migration and cause morphological changes related to damage to the cell cytoskeleton [18]. Interestingly, neutrophils can produce very small amounts of cyanide during chlorination of bacterial cell membranes [19], but this contribution is likely to be negligible in comparison with the high cyanide levels reported herein. Furthermore, the present authors were unable to detect cyanide in eight out of nine sputum samples from CF patients not yet infected by $P$. aeruginosa, although other bacterial pathogens were isolated from the sputum and studies have demonstrated that these subjects will also have a florid airway neutrophilia $[20,21]$. Cyanide was detected in sputum from a very young patient who had no isolated $P$. aeruginosa on routine culture. This patient rarely produces sputum and obtaining the sample was extremely fortunate; however, at present there is no explanation for the cyanide detected. There is the potential that the few culture results available from this individual have been false negatives for $P$. aeruginosa, but this has not been confirmed with more invasive techniques. The patient's clinical progress is being closely monitored continuously and, to date, the patient appears well.

There has been recent debate on the nature of the microbial habitat in the CF lung, particularly with respect to whether conditions are aerobic or anaerobic [3,4]. Understanding lung environmental conditions is clinically important, as the efficacy of several antibiotics is dependent on the chemical milieu, i.e. the aminoglycosides function very poorly under anaerobic conditions [22]. The current findings suggest that a substantial proportion of the $P$. aeruginosa population in the CF lung exists in microaerobic $\left(\mathrm{O}_{2}<5 \%\right)$ rather than aerobic or anaerobic conditions because $P$. aeruginosa can only produce cyanide under strictly microaerobic conditions [8]. Given the oxygen gradient demonstrated in CF mucus, it is also possible that there are 
populations of $P$. aeruginosa dwelling within aerobic or anaerobic regions in the CF lung; however, further studies with other metabolic markers are required to confirm this [4]. This habitat diversity may partly explain the refractory nature of infection, as microbial populations in different physiological states may respond quite differently to therapeutic interventions.

In the relatively small number of sputum samples tested, cyanide levels were similar whether patients were stable or acute, but levels in the latter patients were substantially reduced by a course of intravenous antibiotics. A valid criticism of the study is that the small numbers of patients studied reduced the present authors' ability to detect a difference in sputum cyanide between individuals based on clinical status. Ideally, patients should have been followed from the clinically stable situation into an exacerbation and then been reassessed at the end of treatment, as well as 1 month later when they were once again stable. Such a study clearly needs to be undertaken and correlated with changes in bacterial numbers. The confirmation of a relationship between cyanide and clinical status would open up the development of tools, such as exhaled breath condensates to monitor cyanide as a potential biomarker. Despite the small number of subjects studied, it is interesting to note that the highest cyanide level detected occurred in a stable patient who was the only $P$. aeruginosa-infected patient not receiving any form of anti- $P$. aeruginosa therapy. Chronic suppressive inhaled antibiotic treatment strategies directed against $P$. aeruginosa may, therefore, be partially effective at inhibiting cyanide production, while more aggressive treatment in the present study reduced cyanide in the CF lung to undetectable levels in almost half of the cases. Thus, routine intravenous treatment courses may be of particular benefit in treating this disease. Whether the reduction in cyanide levels following in-patient antibiotics was due to alterations in bacterial numbers or to other nonbactericidal effects was not assessed in this study, but it is worth speculating that the excellent outcomes reported in some countries may be due in part to suppression of cyanide production through regular admissions, irrespective of clinical status, for intravenous antibiotic courses [23, 24].

In vitro data suggests that mucoid $P$. aeruginosa isolates produce more cyanide than nonmucoid laboratory strains [5], but such a relationship was not identified in the present study as all patients were colonised with mucoid strains (data not shown). Whether strains that produce more cyanide have a survival advantage in the CF lung environment or whether the alginate layer in mucoid strains simply increases cyanide output by limiting oxygen diffusion are questions that need to be examined further.

In conclusion, the present study demonstrates large amounts of cyanide in cystic fibrosis sputum. Cyanide is likely to be an important virulence factor employed by Pseudomonas aeruginosa in cystic fibrosis and almost certainly contributes directly to long-term lung damage and impairment of the local host immune system. Of clinical importance, it has been shown that cyanide production can be reduced to very low levels by aggressive antibiotic therapy. The present findings suggest that a substantial proportion of the bacterial population in the cystic fibrosis lung exists under microaerobic rather than anaerobic conditions. Whether cyanide in either sputum or exhaled breath condensates may be a reliable marker of bacterial activity that could be used to assess treatment efficacy in cystic fibrosis requires further study.

\section{REFERENCES}

1 Høiby N. Cystic fibrosis: infection. Schweiz Med Wochenschr 1991; 121: 105-109.

2 Venaille TJ, Ryan G, Robinson BW. Epithelial cell damage is induced by neutrophil-derived, not pseudomonasderived, proteases in cystic fibrosis sputum. Respir Med 1998; 92: 233-240.

3 Yoon SS, Hennigan RF, Hilliard GM, et al. Pseudomonas aeruginosa anaerobic respiration in biofilms: relationships to cystic fibrosis pathogenesis. Dev Cell 2002; 3: 593-603.

4 Worlitzsch D, Tarran R, Ulrich M, et al. Effects of reduced mucus oxygen concentration in airway Pseudomonas infections of cystic fibrosis patients. J Clin Invest 2002; 109: 317-325.

5 Carterson AJ, Morici LA, Jackson DW, et al. The transcriptional regulator $\mathrm{AlgR}$ controls cyanide production in Pseudomonas aeruginosa. J Bacteriol 2004; 186: 6837-6844.

6 Gallagher LA, Manoil C. Pseudomonas aeruginosa PAO1 kills Caenorhabditis elegans by cyanide poisoning. J Bacteriol 2001; 183: 6207-6214.

7 Maresso AW, Deng Q, Pereckas MS, Wakim BT, Barbieri JT. Pseudomonas aeruginosa ExoS ADP-ribosyltransferase inhibits ERM phosphorylation. Cell Microbiol 2007; 9: 97-105.

8 Blumer C, Haas D. Mechanism, regulation, and ecological role of bacterial cyanide biosynthesis. Arch Microbiol 2000; 173: 170-177.

9 Reid DW, Withers NJ, Francis L, Wilson JW, Kotsimbos TC. Iron deficiency in cystic fibrosis: relationship to lung disease severity and chronic Pseudomonas aeruginosa infection. Chest 2002; 121: 48-54.

10 Pin I, Gibson PG, Kolendowicz R, et al. Use of induced sputum cell counts to investigate airway inflammation in asthma. Thorax 1992; 47: 25-29.

11 Goldfarb WB, Margraf $H$. Cyanide production by Pseudomonas aeruginosa. Ann Surg 1967; 165: 104-110.

12 Goldfarb WB, Margraf $H$. Cyanide production by Pseudomonas aeruginosa. Surg Forum 1964; 15: 467-469.

13 Contreras AA, Evans BW, Moncrief JA, Lindberg RB, Villarreal Y, Mason AD Jr. Some aspects of cyanideproducing capabilities of Pseudomonas aeruginosa strains isolated from burned patient infections. J Trauma 1963; 109: 527-533.

14 Musshoff F, Schmidt P, Daldrup T, Madea B. Cyanide fatalities: case studies of four suicides and one homicide. Am J Forensic Med Pathol 2002; 23: 315-320.

15 Brockbank S, Downey D, Elborn JS, Ennis M. Effect of cystic fibrosis exacerbations on neutrophil function. Int Immunopharmacol 2005; 5: 601-608.

16 Church DA, Kanga JF, Kuhn RJ, et al. Sequential ciprofloxacin therapy in pediatric cystic fibrosis: comparative study vs. ceftazidime/tobramycin in the treatment of acute pulmonary exacerbations. The Cystic Fibrosis Study Group. Pediatr Infect Dis J 1997; 16: 97-105.

17 Hampton MB, Zhivotovsky B, Slater AF, Burgess DH, Orrenius S. Importance of the redox state of cytochrome $\mathrm{C}$ 
during caspase activation in cytosolic extracts. Biochem J 1998; 329: 95-99.

18 Fossati G, Moulding DA, Spiller DG, Moots RJ, White MR, Edwards SW. The mitochondrial network of human neutrophils: role in chemotaxis, phagocytosis, respiratory burst activation, and commitment to apoptosis. J Immunol 2003; 170: 1964-1972.

19 Stelmaszynska T. Formation of HCN by human phagocytosing neutrophils-1. Chlorination of Staphylococcus epidermidis as a source of HCN. Int J Biochem 1985; 17: 373-379.

20 Konstan MW, Berger M. Current understanding of the inflammatory process in cystic fibrosis: onset and etiology. Pediatr Pulmonol 1997; 24: 137-142.
21 Armstrong DS, Grimwood K, Carlin JB, et al. Lower airway inflammation in infants and young children with cystic fibrosis. Am J Respir Crit Care Med 1997; 156: 1197-1204.

22 Lewis DA, Jones A, Parkhill J, et al. Identification of DNA markers for a transmissible Pseudomonas aeruginosa cystic fibrosis strain. Am J Respir Cell Mol Biol 2005; 33: 56-64.

23 Doring G, Høiby N. Early intervention and prevention of lung disease in cystic fibrosis: a European consensus. J Cyst Fibros 2004; 3: 67-91.

24 Doring G, Conway SP, Heijerman HG, et al. Antibiotic therapy against Pseudomonas aeruginosa in cystic fibrosis: a European consensus. Eur Respir J 2000; 16: 749-767. 